\title{
Multiresolution Signal Processing on Meshes for Automatic Pathological Shape Characterization
}

\author{
Sylvain Jaume ${ }^{1}$, Matthieu Ferrant ${ }^{2}$, Andreas Schreyer ${ }^{1}$, Lennox Hoyte ${ }^{1}$, \\ Benoît Macq ${ }^{2}$, Julia Fielding ${ }^{1}$, Ron Kikinis ${ }^{1}$, and Simon K. Warfield ${ }^{1}$ \\ 1 Brigham and Women's Hospital and Harvard Medical School, Thorn 329, \\ Department of Radiology, 75 Francis St, Boston, MA, 02115, USA, \\ ${ }^{2}$ Université catholique de Louvain, Telecommunications Laboratory, \\ Place du Levant 2, 1348 Louvain-la-Neuve, Belgium
}

\begin{abstract}
We present a method based on multiresolution signal processing on meshes to create a thickness atlas. We applied this method to construct an atlas of bladder wall thickness. Bladder cancer is associated with increased bladder wall thickness. A thickness atlas helps to detect abnormal thickening in the bladder wall. Extracting inner and outer surface meshes from segmented images, we compute the thickness on the inner surface and map it to a sphere. We average the thickness at each position on the sphere to create a thickness atlas. We then compute Zscore values on the configuration of the patient's bladder to show regions of unusual thickness.
\end{abstract}

\section{Introduction}

Anatomical structures such as bladder wall, prostate wall, heart and cortical gray matter, have a varying thickness. A local thickening of the inner surface can be the first sign of an abnormality and is difficult to be distinguished from the normal variation in thickness. Aligning many surfaces and averaging their thickness values would create a thickness atlas. Areas where thickness is both large and highly different from the atlas would mean a high likelihood of abnormality. While Angenent et al. [1] formulate a conformal mapping with minimal area distortion, we use the wavelets on meshes introduced by Guskov et al. [3] to align surfaces onto a sphere. The benefits are a progressive alignment of the shape from large scale to finer details and a small distortion of the surface parameterization.

\section{Algorithm}

Inner and outer surface meshes are extracted by Marching Cubes [5] from manually segmented and coherently oriented scans. Each vertex of the inner surface gets a thickness value which is the distance to the closest vertex on the outer surface. We then create a Progressive Mesh [4] for each subject model. 
When traversing this representation from coarse to fine, we minimize the curvature of the vertices involved in a vertex split by divided differences [2] and project them to a sphere. Since any position on one sphere can be mapped to another sphere with 2 angles, we can average the thickness at this position on all models to create an atlas. For each subject, a Z-score, $Z(v)=$ $\mid$ thickness $(v)$ - atlas thickness $(v) \mid$ /atlas standard deviation $(v)$, estimates the likelihood of pathological thickness at the vertex $v$.

\section{Results}

We implemented our method in the Visualization Toolkit [6] and applied it on 24 bladder cases to increase the specificity of tumor detection. Figure 1 illustrates our results. Each value of the spherical thickness atlas is an average over all 24 inner surfaces mapped to a sphere. Center images depict one case where a high thickness value matches a high Z-score (white areas). This suspicious area correlates with the abnormality detected by an expert on the CT images (arrow on the right image).

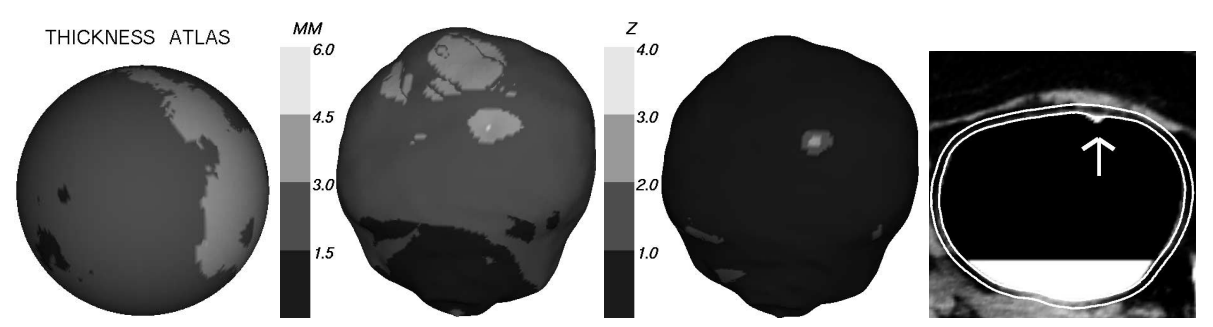

Fig. 1. Thickness atlas from 24 subjects (left), inner surface of one bladder where gray scale codes the thickness (center left) and the Z score (center right), CT slice overlayed with cuts through the inner and outer meshes in white (right), the arrow shows an abnormality detected by an expert.

\section{Discussion and Conclusion}

We presented an algorithm to automatically create a thickness atlas from segmented 3D images of the inner and outer volumes of an anatomical structure. Our method is based on multiresolution signal processing on meshes and introduces small parameterization distortion. The difference between the local thickness in a new scan and the atlas thickness improves the detection of abnormalities. Results on 24 bladder CT scans showed the potentiality of the method for further applications such as designing a thickness atlas of the cortical gray matter.

Acknowledgements: Special thanks to Igor Guskov for many helpful discussions. Sylvain Jaume is working towards a Ph.D. degree with a grant from the Belgian FRIA. This investigation was supported by NIH P41 RR13218, NIH P01 CA67165 and NIH R01 RR11747. 


\section{References}

[1] S. Angenent, S. Haker, A. Tannenbaum, and R. Kikinis. System Theory, Modeling, Analysis, and Control, chapter 20 On area preserving maps of minimal distortion, pages 275-287. Kluwer Academic Publishers, 2000.

[2] I. Guskov. Multivariate subdivision schemes and divided differences. Technical report, Department of Mathematics, Princeton University, 1998.

[3] I. Guskov, W. Sweldens, and P. Schröder. Multiresolution signal processing for meshes. In Computer Graphics (SIGGRAPH 97 Proceedings), pages 325-334, 1997.

[4] Hoppe H. Progressive meshes. In Computer Graphics (SIGGRAPH 96 Proceedings), pages 99-108, 1996.

[5] W.E. Lorensen and H.E. Cline. Marching cubes: a high resolution 3D surface construction algorithm. In Computer Graphics (SIGGRAPH 87 Proceedings), pages 163-169, 1987.

[6] W. Schroeder, K. Martin, and B. Lorensen. The Vizualisation Toolkit: An ObjectOriented Approach to 3D Graphics. Prentice Hall PTR, New Jersey, second edition, 1998. 